\title{
Direct Numerical Simulation in a Lid-Driven Cubical Cavity at High Reynolds Number by a Chebyshev Spectral Method
}

\author{
Emmanuel Leriche ${ }^{1}$
}

Received October 22, 2004; accepted (in revised form) September 2, 2005; Published online January 5, 2006

Direct numerical simulation of the flow in a lid-driven cubical cavity has been carried out at high Reynolds numbers (based on the maximum velocity on the lid), between $1.210^{4}$ and $2.210^{4}$. An efficient Chebyshev spectral method has been implemented for the solution of the incompressible Navier-Stokes equations in a cubical domain. The Projection-Diffusion method [Leriche and Labrosse (2000, SIAM J. Sci. Comput. 22(4), 1386-1410), Leriche et al. (2005, J. Sci. Comput., in press)] allows to decouple the velocity and pressure computation in very efficient way and the simple geometry allows to use the fast diagonalisation method for inverting the elliptic operators at a low computational cost. The resolution used up to 5.0 million Chebyshev collocation nodes, which enable the detailed representation of all dynamically significant scales of motion. The mean and root-mean-square velocity statistics are briefly presented.

KEY WORDS: Chebyshev spectral method; direct numerical simulation; 3D lid-driven cavity.

\section{INTRODUCTION}

Estimates for the attainable turbulent Reynolds number by the method of direct numerical simulation (DNS) have been known for several decades. This estimate is based on the ratio between the largest scales to the finest ones (i.e. Kolmogorov scales), which scales like $R e^{3 / 4}$, where $R e$ is the Reynolds number, and to resolve numerically all the scales, an upper

\footnotetext{
${ }^{1}$ Laboratoire d'Ingénierie Numérique, Institut des Sciences de l'Energie, Section de Génie Mécanique, Faculté des Sciences et Techniques de l'Ingénieur, Ecole Polytechnique Fédérale de Lausanne, Station 9, CH-1015 Lausanne, Switzerland. E-mail: emmanuel.leriche@epfl.ch
} 
bound in term of degrees of freedom (dof) is then given by $R e^{9 / 4}$. The evolution in computer hardware and algorithmic developments makes it now possible to extend the direct numerical simulation to transitional and turbulent flows that are inhomogeneous in all space directions. The present contribution is concerned with the numerical and physical aspects of the direct simulation of incompressible flow within a lid-driven cubical cavity. The fluid is enclosed in a cubical cavity where one wall is moved with a specified velocity. The goal is to reach the highest Reynolds number but in a very simple domain, a cubical cavity and then to study in detail the three-dimensional transitional and turbulent flow properties within the cavity by means of direct simulation at high Reynolds numbers (based on the maximum velocity on the lid), between $1.210^{4}$ and $2.210^{4}$. This simple geometry leads to a very efficient code. The resolution used up to 5.0 million Chebyshev collocation nodes, which enable the detailed representation of all dynamically significant scales of motion. The flow phenomena encountered within such systems are many and poorly understood. To our knowledge, such detailed study of this type of complexity is not available in the scientific literature.

The paper is organized as follows: after Sec. 2 recalling the governing equations, Sec. 3 focuses on decoupling between the velocity and the pressure. The space and time discretizations are very briefly addressed. Section 4 discusses the numerical and physical parameters of the direct numerical simulations in the lid-driven cavity. Section 5 presents some physical aspects of DNS results and some comparison between the three high Reynolds numbers cavity flows. The paper ends with conclusions.

\section{THE GOVERNING EQUATIONS}

The fluid enclosed in the cavity is assumed to be incompressible, viscous, Newtonian and homogeneous. The equation of motion for the fluid inside the cavity is given by the Navier-Stokes equation. The flow domain and naming conventions are given in Fig. 1. The three-dimensional domain, denoted by $\boldsymbol{\Omega}$, is the open interval (]$-h,+h[)^{3}$ and its closure is written as $\overline{\mathbf{\Omega}}$. The Navier-Stokes equations are written in vector notation as

$$
\frac{\partial \mathbf{u}}{\partial t}+(\mathbf{u} \cdot \nabla) \mathbf{u}=-\nabla p+v \Delta \mathbf{u}
$$

with the continuity equation given by

$$
\nabla \cdot \mathbf{u}=0,
$$

where the velocity vector $\mathbf{u}$ has components $(u, v, w) \equiv\left(u_{1}, u_{2}, u_{3}\right)$, and $\mathbf{x} \equiv(x, y, z) \equiv\left(x_{1}, x_{2}, x_{3}\right)$. 


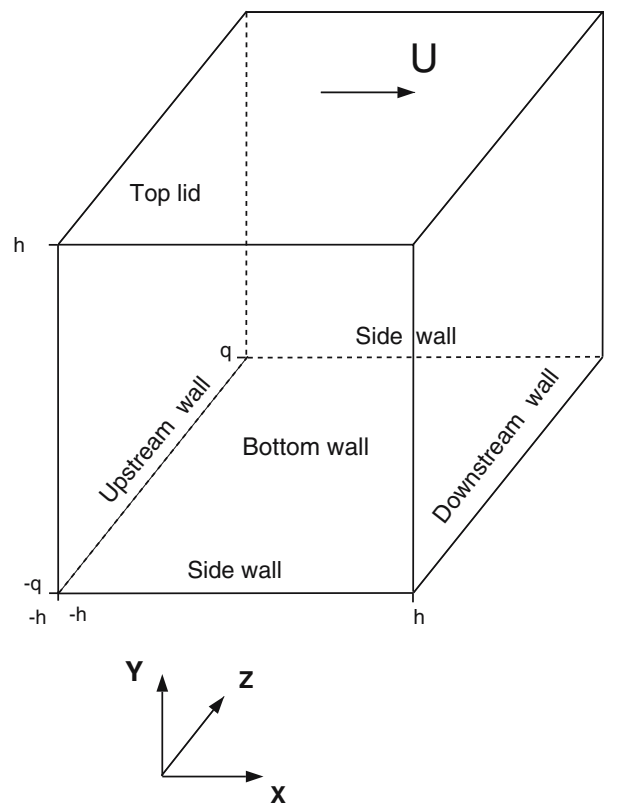

Fig. 1. Sketch of the problem and axes system.

The boundary conditions for the velocity consist in enforcing no-slip at every solid wall except on the moving lid at $y=+h$. The velocity distribution on the lid is given by the following expression

$$
u(x, y=+h, z)=U_{o}\left(1-(x / h)^{n}\right)^{2}\left(1-(z / h)^{n}\right)^{2}
$$

with $n=18$ and where $U_{o}$ is the maximum lid velocity. This lid velocity profile avoids singularity at the top edges of the cavity. The sensitivity of the results to polynomial distribution of the lid velocity profile is discussed in [11] for two-dimensional cavity flow. The Reynolds number is based on the maximum velocity on the lid and is given by $R e=U_{o} 2 h / v$.

\section{NUMERICAL APPROXIMATION}

\subsection{Space Discretization}

The spatial approximation of the equations of motion - the incompressible Navier-Stokes equations - is based on the use of expansions in Chebyshev polynomials along every space direction. All matrices arising from the discretization are cast into the tensor product form with substantial gains in computational efficiency. The collocation method consists of 
exactly enforcing the differential equations, and the boundary conditions, at the Chebyshev-Gauss-Lobatto points $[4,6]$. The velocity and pressure fields belong to the same polynomial space (see next section).

\subsection{How to Enforce the Incompressibility Constraint?}

A number of different methods are available to enforce the incompressibility constraint $[5,9,17]$. The projection-diffusion method introduced in [2] is chosen for its consistency with the continuous space-time problem and for its optimal cost. The pressure is expanded into polynomials of the same order as the velocity, while intermediate velocity results are truncated in a way that enforces continuity without any contamination by spurious pressure modes [10].

A complete numerical analysis of this decoupling method may be found in $[13,14]$ where it is compared with the high-order time splitting scheme proposed by Karniadakis et al. [8].

The unsteady Navier-Stokes problem is solved with a ProjectionDiffusion solver which uncouples the $(\mathbf{u}, p)$ fields independently of any temporal scheme. Section 3.2 in [13] provides all the details about the discrete formulation of the unsteady Stokes problem, which this section relies on. The spatial discretization is based on the usual Chebyshev Gauss-Lobatto collocation method [4,6]. Let $N$ be the polynomial cutoff frequency, where $N+1$ is the number of collocation points, in each space direction. For the sake of conciseness, the equations in this subsection are written as the continuous transposition of the corresponding discrete problem. For instance $\nabla$. stands for the discrete $(N+1)^{3}$ divergence matrix operator and $\mathbf{x} \in \Omega$ means that only the internal nodes are concerned. From Eq. (1), an intermediate divergence free field is introduced, the acceleration $\mathbf{a}$,

$$
\mathbf{a}=\frac{\partial \mathbf{u}}{\partial t}-v \Delta \mathbf{u} \quad \text { for } \mathbf{x} \in \bar{\Omega}
$$

The problem is solved through two steps:

First Step: the pressure is evaluated from

$$
\begin{aligned}
\mathbf{a}+\nabla p & =-\mathbf{f} \quad \text { for } \mathbf{x} \in \Omega_{i}, \quad(i=1,2,3), \\
\nabla \cdot \mathbf{a} & =0 \text { for } \mathbf{x} \in \bar{\Omega}, \\
\mathbf{a} \cdot \mathbf{n} & =\left(\frac{\partial \mathbf{u}}{\partial t}+\nu \nabla \times \nabla \times \mathbf{u}\right) \cdot \mathbf{n} \text { for } \mathbf{x} \in \partial \Omega,
\end{aligned}
$$


where

$$
\begin{aligned}
& \left.\Omega_{1}=\right]-h,+h[\times[-h,+h] \times[-h,+h], \\
& \left.\Omega_{2}=[-h,+h] \times\right]-h,+h[\times[-h,+h], \\
& \left.\Omega_{3}=[-h,+h] \times[-h,+h] \times\right]-h,+h[
\end{aligned}
$$

for the respective components of Eq. (5). The source term $\mathbf{f}$ stands for the non-linear advective terms (treated explicitly in time, see next section). As explained in [13], the $-\nabla \times \nabla \times \mathbf{u}$ solenoidal term in the normal boundary condition (7) is chosen instead of $\Delta \mathbf{u}$ in order to preserve the ellipticity of the Stokes problem. The irrotational part $\nabla(\nabla \cdot \mathbf{u})$ is dropped in accordance with Eq. (2). The first step (5)-(7) allows the computation of the pressure through the solution of a Darcy type problem [1], assuming that the r.h.s. of the normal boundary condition (7) is known (see below). The first step (5)-(7) is recast into a quasi-Poisson operator taking into account the normal boundary condition (7).

Second Step: the velocity is evaluated through the diffusion step

$$
\begin{array}{rlrl}
\frac{\partial \mathbf{u}}{\partial t}-\Delta \mathbf{u} & =\mathbf{a} & & \text { for } \mathbf{x} \in \Omega, \\
\mathbf{u}=0 & & \text { for } \mathbf{x} \in \partial \Omega .
\end{array}
$$

\subsection{Time Integration}

The time discretization of the Navier-Stokes equations is based on a second-order backward Euler differentiation formula. The viscous terms are treated implicitly whereas the non-linear terms are advanced explicitly in time by a second-order extrapolation scheme. They are evaluated from their skew-symmetric form. The normal boundary conditions (7) is found by a second-order extrapolation scheme. The overall accuracy is second-order in time and the scheme is subject to (CFL) restrictions in the time step due to the explicit advancement of the non-linear terms (see next section).

\section{PHYSICAL AND COMPUTATIONAL PARAMETERS}

The numerical simulations in the cubical cavity at Reynolds numbers of 12000 (resp. 18000 and 22000) have been performed on the NEC-SX4/5 at Swiss Center for Scientific Computing (CSCS-Manno) with a resolution of $129^{3}$ (resp. $169^{3}$ ) collocation points. The main computational and physical parameters are reported in Table I. The very simple geometry of the 
Table I. Main Physical and Numerical Parameters for Direct Numerical Simulations at Reynolds Numbers 12000, 18000, and 22000

\begin{tabular}{llll}
\hline $\begin{array}{l}\text { Domain size (x,y,z) } \\
R e=U_{o} 2 h / v\end{array}$ & $\begin{array}{l}2 \mathrm{~h}, 2 \mathrm{~h}, 2 \mathrm{~h} \\
\text { Nb. Chebyshev }\end{array}$ & $\begin{array}{l}2 \mathrm{~h}, 2 \mathrm{~h}, 2 \mathrm{~h} \\
18000\end{array}$ & $\begin{array}{l}2 \mathrm{~h}, 2 \mathrm{~h}, 2 \mathrm{~h} \\
22000\end{array}$ \\
$\begin{array}{l}\text { Polynomials (x,y,z) } \\
\text { Grid Resolution min }\end{array}$ & $129^{3}$ & $169^{3}$ & $169^{3}$ \\
Grid Resolution max & & & \\
Time step $\left(h / U_{o}\right)$ & $0.000301 \mathrm{~h}$ & $0.000175 \mathrm{~h}$ & $0.000175 \mathrm{~h}$ \\
CFL max & $0.0245 \mathrm{~h}$ & $0.0187 \mathrm{~h}$ & $0.0187 \mathrm{~h}$ \\
Computers (NEC) & 0.0025 & 0.0015 & 0.00125 \\
Sustained Gflop rate $($ Peak) & 0.29 & 0.26 & 0.23 \\
Cpu(sec)/time step & $\mathrm{SX} 4$ & $\mathrm{SX} 5$ & $\mathrm{SX} 5$ \\
Cpu(sec)/(time step*node) & $1.8(2.0)$ & $6.5(8.0)$ & $6.5(8.0)$ \\
Total integration time $\left(h / U_{o}\right)$ & 20.625 & 17.00 & 17.00 \\
Sampling rate $\left(h / U_{o}\right)$ & $9.61 \times 10^{-6}$ & $3.5 \times 10^{-6}$ & $3.5 \times 10^{-6}$ \\
Nb. of samples & 1000 & 1400 & 3500 \\
Size of one sample $(\mathrm{Mb})$ & 0.25 & 0.1275 & 0.10625 \\
Total data held in store & 4000 & 10000 & 30000 \\
& 68.7 & 154.5 & 154.5 \\
\hline
\end{tabular}

cavity allows to write a very efficient code based on solution of elliptic operators using the fast diagonalisation method [7, 15]. The code is then highly vectorized and could reach more than $80 \%$ of the peak performance of a single NEC processor. The time steps were chosen in order that the corresponding CFL number given by $\sum_{i=1}^{3}\left|\frac{u_{i} \Delta t}{\Delta x_{i}}\right|$ are $10 \%$ below the CFL limit given in Table I. $\Delta x_{i}$ is the distance between two neighbouring collocation points in the $x_{i}$ direction. The $\mathrm{CFL}_{\mathrm{MAX}}$ turns out to decrease when increasing the mesh size but also when increasing the Reynolds number.

The spatial accuracy of the simulations is gauged by monitoring the evolution with time of the absolute values of the ratios of the lowest Chebyshev mode to that of the highest for each component of the velocity field in the spectral space. They did not exceed $10^{-3}$. This is stricter criteria than those based on statistics such as spectra. The asymptotic behavior of the three velocity components near each boundary was also verified. An additional check was the balance of the terms in the equation for the turbulent kinetic energy-not shown here.

The databases are generated by storing the three-dimensional velocity and pressure fields in order to get first- and second-order statistics taken on meaningful sample. The sizes of those databases are of the order of 250 Gbytes $(R e=12000), 1.7$ Tbytes $(R e=18000)$ and 5.4 Tbytes $(R e=22000)$. It turns out that the statistics for the case of $R e=22000$ 
require much more longer sample than the one at $R e=18000$. The total integration time for the simulations reported in Table I leads to first- and second-order velocity statistics approximately symmetric about the midplane $z / h=0$, the mid-plane being a plane of statistical symmetry. Both transient and steady-in-the-mean states of the flow possess long time scales requiring long integration times.

\section{SOME DNS RESULTS}

The most exhaustive quantitative experimental data for the lid driven cavity flow may be found in [16]. Measurements (mean and root-meansquare $(r m s)$ ) on the vertical and horizontal centerlines in the mid-plane are reported at different Reynolds numbers up to 10000 . The experimental data at the highest Reynolds number of 10000 provide a fair agreement with the simulation at the lowest Reynolds number of 12000 [12]. Nonetheless, this comparison is limited to the mid-plane.

The first- and second-order velocity statistics have been computed for the three Reynolds numbers. The effects of Reynolds number on the driven cavity flow will be preliminary discussed here. The kinetic energy $K$ contained in the thin viscous layer of fluid close to the lid is successively transferred into the cavity flow by viscous diffusion. The total kinetic energy is shown to decrease with the Reynolds number (Fig. 2) in accordance with the estimate $K \approx U_{o}^{2}(2 h)^{3} R e^{-1 / 2}$ given in [12].

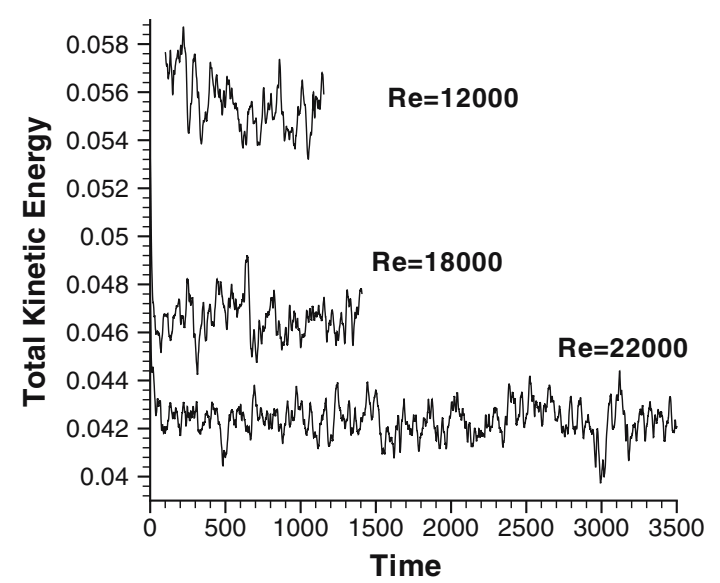

Fig. 2. Time history of the total kinetic energy for Reynolds numbers 12000, 18000, and 22000 . 
The most dominant features of the mean flow are the large-scale recirculation which spans the cavity, the coexistence of fast- and slowflowing regions near the moving lid, the presence of wall jet (parallel to the downstream, bottom and, at higher Reynolds numbers, the upstream vertical wall), of jets impingement on the bottom and upstream wall, and of corner spiralling vortices [12]. Figure 3 shows contours of the $\mathrm{u}$ and $\mathrm{v}$ mean fields in the mid-plane of the cavity. The viscous layer close to the lid and the downstream wall jet are getting thinner as the Reynolds increases. The enlargements of the downstream bottom corner clearly show that the size of corner vortices decrease as the Reynolds increases.

Contours of the rms (root-mean-square) of $u, v$ and $w$ in the midplane near the downstream bottom corner are shown in Fig. 4. The rms increase with the Reynolds number (Table II), faster for $u$-rms and slower for $v$-rms.
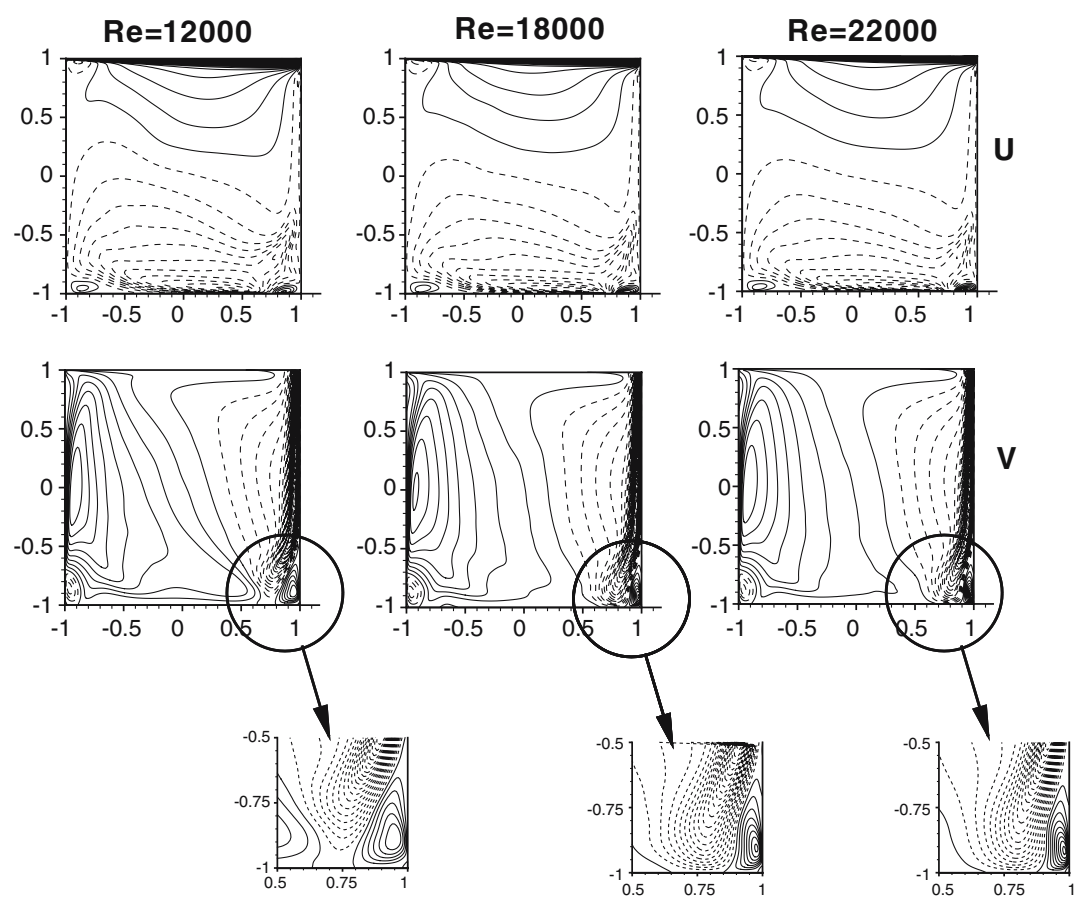

Fig. 3. Contours of the mean velocity field $u$ and $v$ in the mid-plane $z=0$ (coordinates $(x / h, y / h))$. Top figures: equidistant mean velocity $u$ contours; maximum contour level 1.0, minimum contour level -0.21 , interval of 0.019 . Bottom figures: equidistant mean velocity $v$ contours; maximum contour level 0.1 , minimum contour level -0.7 , interval of 0.0125 . 

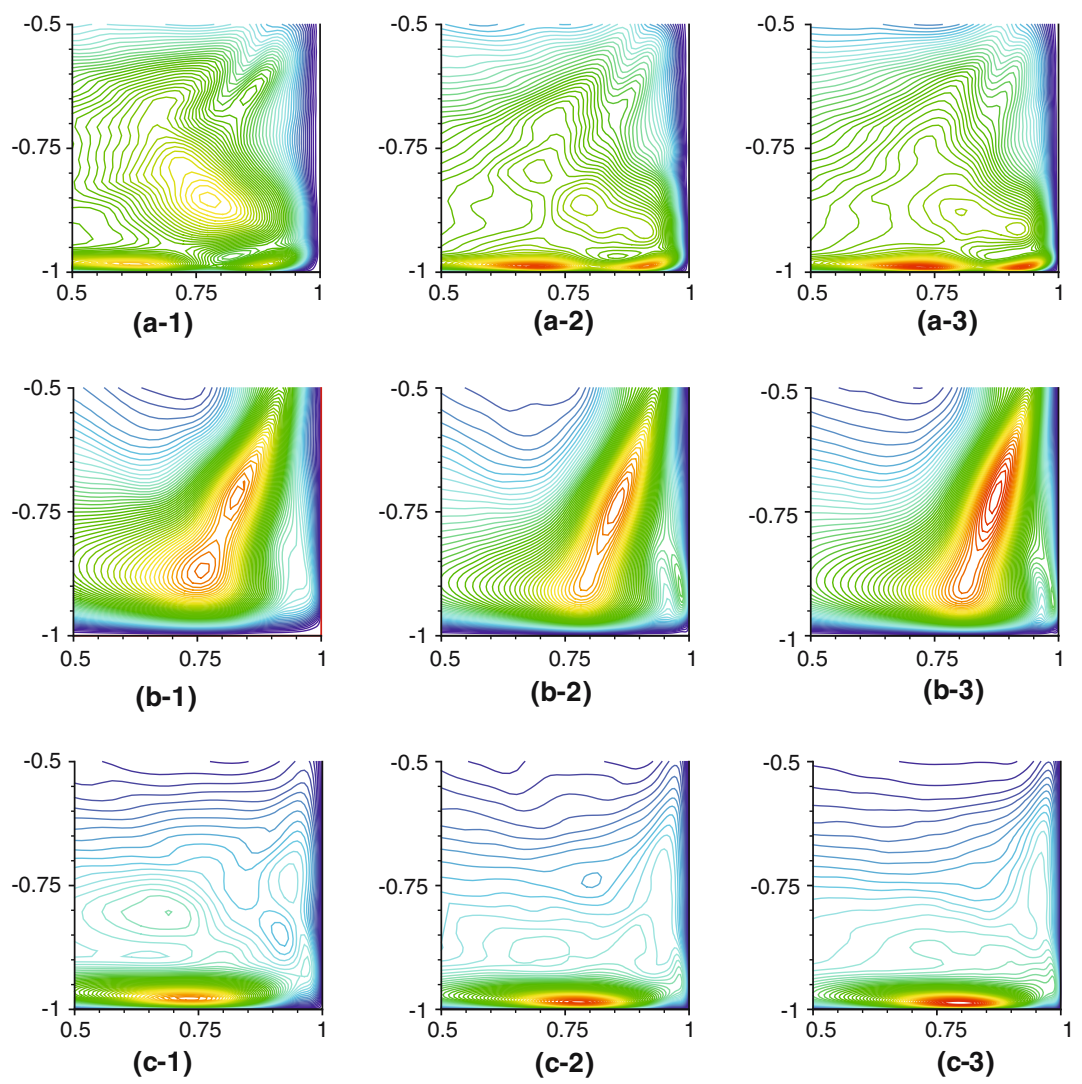

Fig. 4. Contours of the rms of $u$ (a), $v$ (b), and $w$ (c) for the Reynolds numbers 12000 (1), 18000 (2), and 22000 (3) in downstream bottom corner of the mid-plane $z=0$ (coordinates $(x / h, y / h))$. (a) equidistant $u$-rms contours; maximum contour level: 0.085 , interval of 0.0013 . (b) equidistant $v$-rms contours; maximum contour level: 0.16 , interval of 0.0025 . (c) equidistant $w$-rms contours; maximum contour level: 0.17 , interval of 0.0027 .

Table II. Maximum rms (root-meansquare) Values of $u, v$, and $w$ in the MidPlane at Reynolds Numbers 12000, 18000 and 22000

\begin{tabular}{llll}
\hline Reynolds & 12000 & 18000 & 22000 \\
\hline$u$-rms & 0.07 & 0.082 & 0.085 \\
$v$-rms & 0.146 & 0.147 & 0.159 \\
$w$-rms & 0.15 & 0.164 & 0.175 \\
\hline
\end{tabular}


An analysis of the mean and fluctuating fields near the downstream bottom corner shows that the turbulence is generated by the impact of the flow descending near the downstream vertical wall (downstream wall jet). The jet impact on bottom wall can be easily determined by looking at the pressure distribution on the bottom wall — not shown here. The peaks of pressure at the impact are getting narrower as the Reynolds increases. Along the downstream vertical wall, the flow is not turbulent but highly chaotic and near the bottom wall, the rms are found to reach their maximum values at the jets impact and in their vicinity, Fig. 4.

\section{CONCLUSION AND FUTURE WORK}

Direct numerical simulations using a Chebyshev collocation method have been performed for the lid-driven cavity flow at high Reynolds number. The instantaneous three-dimensional velocity and pressure fields have been stored in databases for three Reynolds numbers (12000, 18000, and 22000) and first- and second order velocity statistics have been computed on long integration time to capture long time scales of the cavity flow. The mean momentum budgets are currently investigated and the leading terms in these balances are examined. The Reynolds stress budgets have been computed and the statistics for the distribution of energy between the various components will be discussed.

The databases are currently used in the framework of the large eddy simulation to validate filtering approaches and a priori/a posteriori simulation tests. Preliminary results may be found in [3].

\section{ACKNOWLEDGMENTS}

The author would like to thank Prof. M.O. Deville for helpful discussions. The computing resources were made available by CSCS, Manno, Switzerland.

\section{REFERENCES}

1. Azaiez, M., Bernardi, C., and Grundmann, M. (1995). Spectral method applied to porous media equations. East-West J. Numer. Math., 2, 91-105.

2. Batoul, A., Khallouf, H., and Labrosse, G. (1994). Une méthode de résolution directe (Pseudo-Spectrale) du problème de Stokes 2D/3D instationnaire. Application à la Cavité Entrainée Carrée. C.R. Acad. Sci. Paris. 319(I), 1455-1461.

3. Bouffanais, R., Deville, M. O., Fischer, P. F., Leriche, E., and Weill, D. (2005). Direct and large eddy simulation of incompressible viscous fluid flow by the spectral element method. J. Sci. Comput. DOI: 10.1007/s10915-005-9039-7. 
4. Canuto, C., Hussaini, M. Y., Quarteroni, A., and Zang, T. A. (1988). Spectral Methods in Fluid Dynamics, Springer Series in Computational Physics, Springer-Verlag, New-York.

5. Deville, M. O., Fischer, P. F., and Mund, E. H. (2002). High-Order Method for Incompressible Fluid Flow, Cambridge University Press, Cambridge.

6. Gottlieb, D., and Orszag, S. A. (1977). Numerical Analysis of Spectral Methods: Theory and Applications, SIAM-CBMS, Philadelphia.

7. Haldenwang, P., Labrosse, G., Abboudi, S. A., and Deville, M. (1984). Chebyshev 3D spectral and 2D pseudospectral solvers for the helmholtz equation. J. Comput. Phys. 55, $115-128$.

8. Karniadakis, G. E. M., Israeli, M., and Orszag, S. A. (1991). High-order splitting methods for the incompressible Navier-Stokes equations. J. Comput. Phys. 97, $414-443$.

9. Karniadakis, G. Em., and Sherwin, S. J. (1999). Spectral/hp element methods for CFD, Oxford University Press, New-York.

10. Labrosse, G. (1993). Compatibility conditions for the Stokes system discretized in 2D cartesian domains. Comput. Meth. Appl. Mech. Eng. 106, 353-365.

11. Leriche, E. (1999). Direct Numerical Simulation of a Lid-Driven Cavity Flow by a Chebyshev Spectral Method, Ph.D thesis, no:1932, Ecole Polytechnique Fédérale de Lausanne, Lausanne.

12. Leriche, E., and Gavrilakis, S. (2000). Direct numerical simulation of the flow in a liddriven cubical cavity. Phys. Fluids 12(6), 1363-1376.

13. Leriche, E., and Labrosse, G. (2000). High-order direct Stokes solvers with or without temporal splitting: Numerical investigations of their comparative properties. SIAM J. Sci. Comput. 22(4), 1386-1410.

14. Leriche, E., Perchat, E., Labrosse, G., and Deville, M. O. (2005). Numerical evaluation of the accuracy and stability properties of high-order direct Stokes solvers with or without temporal splitting. To appear in J. Sci. Comput.

15. Lynch, R. E., Rice, J. R., and Thomas, D. H. (1964). Direct solution of partial difference equations by tensor product methods. Numerishe Mathematik 6, 185-199.

16. Prasad, A. K., and Koseff, J. R. (1989). Reynolds number and end-wall effects on a liddriven cavity flow. Phys. Fluids 1(2), 208-218.

17. Schumack, M., Schultz, W., and Boyd, J. (1991). Spectral method solution of the Stokes equations on nonstaggered grids. J. Comput. Phys. 94, 30-58. 